\title{
A Nonstationary Poisson View of Internet Traffic
}

\author{
Thomas Karagiannis, Mart Molle, Michalis Faloutsos \\ Department of Computer Science \& Engineering \\ University of California, Riverside \\ \{tkarag,mart,michalis\}@cs.ucr.edu
}

\author{
Andre Broido \\ CAIDA, SDSC \\ University of California, San Diego \\ broido@caida.org
}

\begin{abstract}
Since the identification of long-range dependence in network traffic ten years ago, its consistent appearance across numerous measurement studies has largely discredited Poissonbased models. However, since that original data set was collected, both link speeds and the number of Internet-connected hosts have increased by more than three orders of magnitude. Thus, we now revisit the Poisson assumption, by studying a combination of historical traces and new measurements obtained from a major backbone link belonging to a Tier 1 ISP. We show that unlike the older data sets, current network traffic can be well represented by the Poisson model for sub-second time scales. At multi-second scales, we find a distinctive piecewise-linear non-stationarity, together with evidence of long-range dependence. Combining our observations across both time scales leads to a time-dependent Poisson characterization of network traffic that, when viewed across very long time scales, exhibits the observed long-range dependence. This traffic characterization reconciliates the seemingly contradicting observations of Poisson and long-memory traffic characteristics. It also seems to be in general agreement with recent theoretical models for large-scale traffic aggregation.
\end{abstract}

\section{INTRODUCTION}

Does the observed long-range dependence make Poissonbased models obsolete? This is a key question for our work.

During the last decade, there has been ample evidence of long-range dependence, scaling phenomena and heavy tailed distributions in various aspects of network behavior. Specifically, it has been observed that packet interarrival times are described by marginal distributions with heavier tail than that of the exponential. Furthermore, networking series such as the aggregate number of packets and bytes in time, have been shown to exhibit correlations over large time scales (i.e., long-range dependence) and self-similar scaling properties. These findings resulted in invalidating the traditionally used assumptions in modeling and simulations, namely that packet arrivals are Poisson and packet sizes and interarrival times are mutually independent.

The first empirical evidence of self-similar characteristics in local area network traffic were presented in the pioneering work in [28]. The authors performed a rigorous statistical analysis of Ethernet traffic measurements and were able

This work was supported by the NSF CAREER grant ANIR 9985195, and DARPA award NMS N660001-00-1-8936, and NSF grant IIS-0208950 TCS Inc., and DIMI matching fund DIM00-10071. and DARPA award FTN F3060201-2-0535 to establish its self-similar nature. Similar observations were presented for wide area Internet traffic in [36], where it was also shown that interarrival times are described by heavy tailed distributions. The origins of self-similarity in Internet traffic have been mainly attributed to heavy tail distributions of transfer sizes [10] [44] [34]. Apart from long-range dependence, it has been observed that Internet traffic presents complex scaling and multifractal characteristics that were usually associated with Round-Trip Time (RTT) delay [16] [41] [47] [37] [19]. In addition, studies have argued whether or not TCP congestion control contributes to the observed scaling [42] [43] [29].

We believe it is time to reexamine the Poisson traffic assumption in relation to the traffic carried within the Internet core. Long before the identification of self-similar characteristics in Internet traffic, Poisson packet arrivals and the independence assumption were widely used as the basis for network modeling and analysis [24]. Despite the tendency of the community to discard Poisson models as being overly simplistic, they can be used to represent the limiting behavior of an aggregate traffic flow created by multiplexing large numbers of independent sources [23] [39]. Thus, given the tremendous growth of the Internet backbone in recent years, we anticipate that any peculiarities due to individual flows might cancel out due to the vast number of different multiplexed flows. Thus, we revisit the Poisson assumption subject to the following caveats:

Aggregated traffic vs. individual flows: We consider the combined packet arrival stream generated by all sources, rather than focusing on the subset of packets generated by a single source. Because of our focus on the highly-multiplexed Internet core, such primary performance metrics as packet delays and buffer occupancies should be insensitive to the details of an individual flow.

Idle periods vs. back-to-back packets: It is well known that the packet interarrival time distribution may deviate from the Poisson model for very small values because of multiple-packet deterministic sequences. In our case, the primary cause will be "busy periods" at the upstream router, which transmits back-toback packets until it manages to empty the queue. In other studies, fixed delay transaction-oriented protocols like NFS, and processing time bottlenecks in the hosts have been identified as the causes for particular "spikes" appearing in the interarrival time distribution [18]. Such short-range artifacts can be 
incorporated into the Poisson model as "packet trains" [20].

The effect of the scale of observation: For moderately loaded network resources, the system will rapidly respond to any short-range transients in the load. Thus, by measuring the system's behavior across larger time scales, individual transient events become less significant relative to the long-term averages, allowing us to determine the steady-state behavior of the system. Eventually, however, we may reach a point, where further increases in the length of the measurement period can actually hurt us because of the presence of nonstationarity. More specifically, long-term nonstationarity can interfere with variance calculations because the global average across very large time scales may drift very far away from the short-term average. To see why such discrepancies might make things obscure, rather than just different, we offer the following analogy.

Consider the problem of determining correlation between the motions of two insects wandering randomly around a small garden. To an observer in the garden who watches the two bugs, their motions might appear completely independent and uncorrelated. However, to an observer watching the two bugs from outer space, the motions of the two bugs appear almost perfectly correlated, since they are never more than a few inches apart as they traverse a daily rotation of the earth around its axis, which is itself embedded in an annual orbit of the earth around the sun. Clearly, estimating the motions of the two bugs relative to some "average" derived from celestial-scale measurements is not appropriate for solving this problem! Similarly, we should not try to normalize all network measurements relative to some far away global long-term average value that the system may never reach within the time scales relevant to the calculation of its primary performance metrics.

In this paper, we show and explain the coexistence of Poisson distributions and long-range dependence in traces from the MFN and WIDE backbones. Traffic can be viewed from two different perspectives: Multifractal scaling as described in [16] [15] [47] or nonstationary Poisson modeling. More specifically, our findings can be summarized in the following points:

- Packet arrivals appear Poisson at sub-second time scales: The packet interarrivals follow an exponential distribution. In addition, packet sizes and interarrival times appear uncorrelated. These observations agree with traditional modeling of network arrivals as Poisson processes.

- Internet traffic appears nonstationary at multi-second time scales: We demonstrate that traffic oscillates around a global mean, in a piecewise linear manner.

- Internet traffic exhibits long-range dependence (LRD) at scales of seconds and above: In agreement with previous findings, we observe that Internet traffic exhibits LRD properties at large time-scales.

Our work in perspective. Our work attempts to reconciliate the seemingly contradictory observed phenomena of LRD and Poisson packet arrivals. Our study does not contradict the observations of previous studies. For example, we confirm both the identification of LRD behavior at large time-scales and the different scaling behavior of traffic at smaller time-scales.

In addition, the issue of LRD modeling versus nonstationarity was also raised in earlier network studies ${ }^{1}$ regarding the famous Bellcore traces [11] [12] [14]. However, in the Bellcore traces, the arrival process clearly deviates from Poisson, which is not the case in our backbone traces. Given this difference in the behavior, we find important to revisit this issue.

Finally, what do our observations mean to a practitioner? Our traces suggest that Poisson models should not be abandoned especially in the Internet core with high speeds, and high levels of traffic multiplexing. For example, simulations may get sufficiently accurate results by varying the arrival rate of a Poisson process.

The rest of this paper is structured as follows: Section II gives a brief description of self-similarity and long-range dependence. Section III describes our traces. Sections IV and V demonstrate the Poisson and nonstationary nature of Internet traffic. Section VI presents the scaling behavior of backbone traffic. Section VII concludes the paper.

\section{DEFINITIONS}

This section briefly presents concepts that will be used in the paper and a brief description of long-range dependence and self-similarity.

We extensively use the complementary cumulative distribution function $(C C D F)$ throughout this paper. The CCDF is defined as, $F^{c}(t)=1-F(t)$, where $F(t)$ is the cumulative distribution function (CDF). The CCDF of the exponential distribution with mean $1 / \lambda$ is

$$
F^{c}(t)=e^{-\lambda t}, t \geq 0 .
$$

Long-range dependence measures the memory of a process. Intuitively, distant events in time are correlated. This correlation is captured by the autocorrelation function $(A C F), \rho(k)$, which measures the similarity between a series $X_{t}$, and a shifted version of itself, $X_{t+k}$ :

$$
\rho(k)=\frac{E\left[\left(X_{t}-\mu\right)\left(X_{t+k}-\mu\right)\right]}{\sigma^{2}}
$$

where $\mu, \sigma$ are the sample mean and standard deviation respectively. If a stationary process has nonsummable autocorrelation function [4], that is $\sum_{k=1}^{\infty} \rho(k)=\infty$, then this process is longrange dependent. Intuitively, there is non-zero correlation even for infinitely large $k$. On the contrary, short-range dependence is characterized by quickly decaying correlations (e.g., ARMA processes).

Self-similarity describes the phenomenon where certain properties are preserved irrespective of scaling in space or time. A stochastic process $X(t)$ is self-similar if $X(a t)=a^{H} X(t)$,

\footnotetext{
${ }^{1}$ The same question also appears in all disciplines where LRD modeling is applied, such as finance [25].
} 
TABLE I

OC48 TRACES ANALYZED.

\begin{tabular}{|c|c|c|c|c|c|c|}
\hline & \multicolumn{2}{|c|}{ August 2002, $14(11: 00-11: 20)$} & \multicolumn{2}{|c|}{ January 2003, $15(10: 00-10: 10)$} & \multicolumn{2}{|c|}{ April 2003, 24 (00:00-00:20 } \\
\hline & Direction 0 & Direction 1 & Direction 0 & Direction 1 & Direction 0 & Direction 1 \\
\hline Bytes & $58.2 \mathrm{G}$ & $92 \mathrm{G}$ & $21 \mathrm{G}$ & $24 \mathrm{G}$ & $14.8 \mathrm{G}$ & $17.4 \mathrm{G}$ \\
\hline Packets & $140.8 \mathrm{M}$ & $145 \mathrm{M}$ & $41.2 \mathrm{M}$ & $34.6 \mathrm{M}$ & $28.8 \mathrm{M}$ & $42.5 \mathrm{M}$ \\
\hline Mean Rate & 333Mbps & 612Mbps & 318Mbps & 278Mbps & 98Mbps & 116Mbps \\
\hline Mean Flows/sec & 18,590 & 19,118 & 16,193 & 18,783 & 8,712 & 9,494 \\
\hline \% TCP bytes (packets) & $89.7(58.7)$ & $97.2(92.6)$ & $91.4(88.8)$ & $96.1(91.1)$ & $96.7(91)$ & $95(86.6$ \\
\hline \% UDP bytes (packets) & $9.8(40.6)$ & $2.2(6.7)$ & $4.9(8.1)$ & $3.2(6.9)$ & $3.1(8)$ & $4(12.7)$ \\
\hline
\end{tabular}

where $\mathrm{H}$ is the self-similarity parameter, namely the Hurst exponent.

In time-series, second-order self-similarity describes the property that the correlation structure (ACF) of a time-series is preserved irrespective of time aggregation. Simply put, the autocorrelation function of a second-order self-similar time-series is the same in either coarse or fine time scales. The aggregated process $X^{(m)}(k)$ is defined as follows:

$$
X^{(m)}(k)=\frac{1}{m} \sum_{i=(k-1) m+1}^{k m} X_{i}, \quad k=1,2, \ldots,\left[\frac{N}{m}\right] .
$$

A stationary process $X_{t}$ is asymptotically second-order selfsimilar [35], if

$$
\lim _{k \rightarrow \infty} \rho(k)=\frac{1}{2}\left[(k+1)^{2 H}-2 k^{2 H}+(k-1)^{2 H}\right] .
$$

Second-order self-similar processes are characterized by a hyperbolically decaying autocorrelation function and are extensively used to model long-range dependent processes.

The notion of stationarity refers to the stability of the behavior. Most traffic models assume, explicitly or implicitly, a stable behavior over a period of time. The stationarity assumption is critical when self-similar behavior is studied, since nonstationarity can lead to misidentification of self-similarity. Furthermore, all Hurst exponent estimation methodologies assume a stationary time-series and their estimates are quite sensitive to the existence of nonstationarities.

While the concepts of self-similarity and long-range dependence are often used interchangeably in the literature, they are not equivalent. Although second-order self-similarity usually implies long-range dependence (i.e., nonsummable ACF), the reverse is not necessarily true. In addition not all self-similar processes are long-range dependent (e.g., Brownian motion).

\section{DATA DESCRIPTION}

We use three types of traces for our study: a) Internet backbone traces from an OC48 link, b) traces from the WIDE backbone maintained by the MAWI Working Group Traffic Archive and the WIDE project [2] [9], and c) the "well-known" $B C$ pAug89 and $L B L-P K T-4$ traces [1] which were analyzed in [28] and [36] respectively.
The WIDE backbone traces were captured in a trans-Pacific 100Mbps link. They are 15 minute traces taken daily at 14:00 local time (JST). We use traces from June 2003. The BCpAug89 trace was taken at 11:25 (EDT) on August 29, 1989 on an Ethernet at the Bellcore Morristown Research and Engineering facility. It consists of $1,000,000$ packets (approximately 3142.82 seconds). Finally, the LBL-PKT-4 was captured on January 21, 1994, 14:00-15:00 (PST) at Lawrence Berkeley Laboratory (approximately 1.3M packets).

Our primary focus in this work is on the OC48 backbone traces. These were taken on CAIDA monitor located at a SONET OC48 (2.5 Gbps) link that belongs to MFN, a US Tier 1 Internet Service Provider (ISP).

The traces were collected by Linux-based monitor with Dag 4.11 network cards and packet capture software originally developed at the University of Waikato [31] and currently produced by Endace [13]. The nominal resolution of the Dag 4.11 card timestamp is $15 \mathrm{~ns}$. A number of technological factors prevent Dag from reaching corresponding precision. The largest source of uncertainty is Sonet overhead that makes interpretation of time differences under $1 \mu s$ problematic [31]. The traffic is monitored in both directions. The captured packet traces contain 44 bytes of each packet, enough to include the IP and TCP/UDP headers.

We analyze three different backbone traces captured in August 2002, January 2003 and April 2003. Table I shows the specific dates these traces were collected, the mean rate in Mbps, the average number of flows per second, as well as the total number of packets and bytes for each trace. In general, the link in question is overprovisioned. With the exception of direction 1 of the August trace that has average utilization of approximately $24 \%$, the utilization for the rest of the traces is rarely over $15 \%$. The number of active flows per second varies between 15,000 - 20,000 for the January and August traces, and between 8,000 - 12,000 for the April traces.

TCP accounts for the vast majority of the traffic. Approximately $95 \%$ of the bytes and $90 \%$ of the packets are transfered with TCP. However, UDP represents a significant portion of the total traffic for direction 0 of the August dataset. In this trace, there is a UDP flood which significantly increases the percentage of UDP packets.

Overall, the traffic on this measured backbone link multiplexes a wide variety of diverse sources, since the Tier 1 ISP 

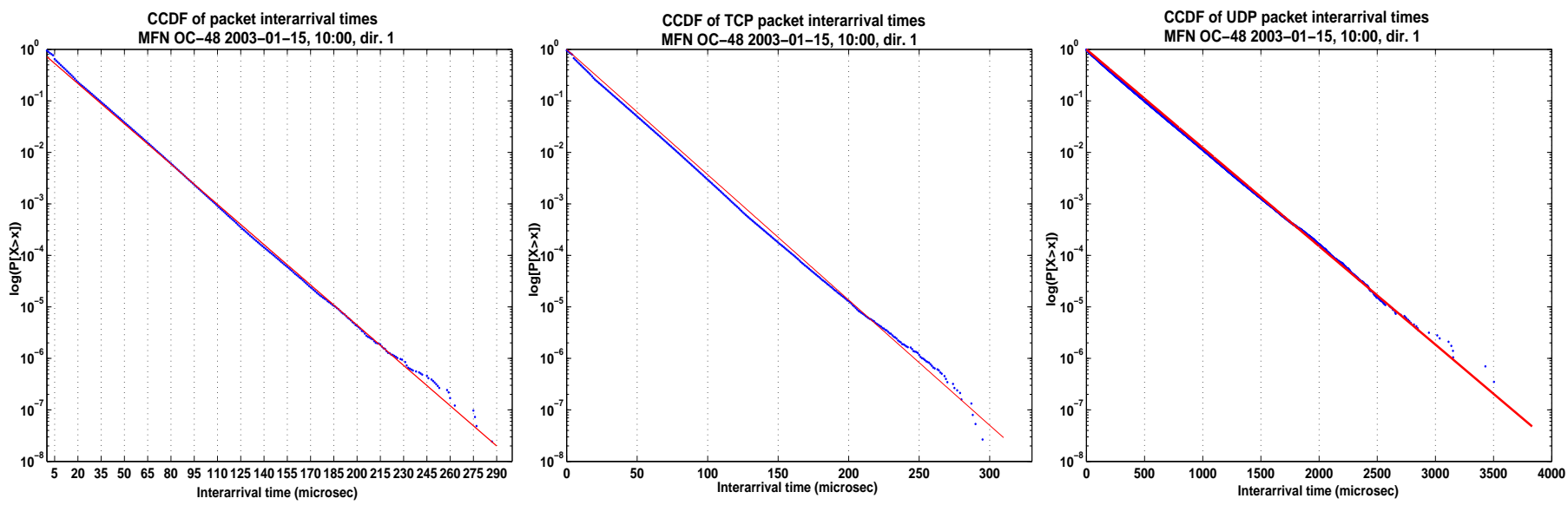

Fig. 1. CCDF of packet interarrival times for OC48 link traces, for aggregate (total), TCP and UDP traffic. The distributions can be well approximated by an exponential distribution.

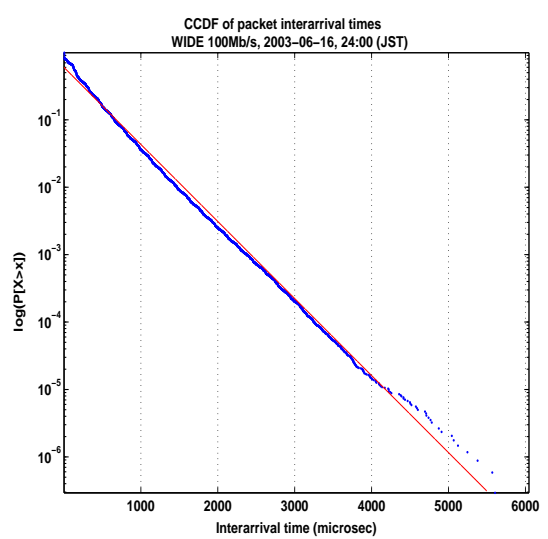

(a) CCDF of packet interarrival times for WIDE backbone (June 16, 2003).

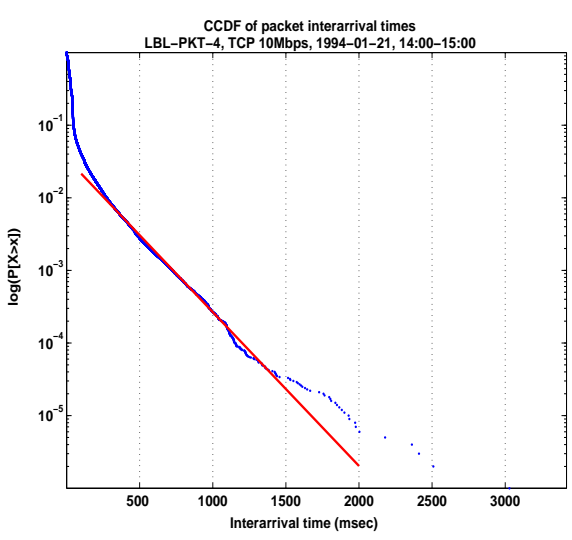

(b) CCDF of packet interarrival times for the LBL-PKT-4 trace.

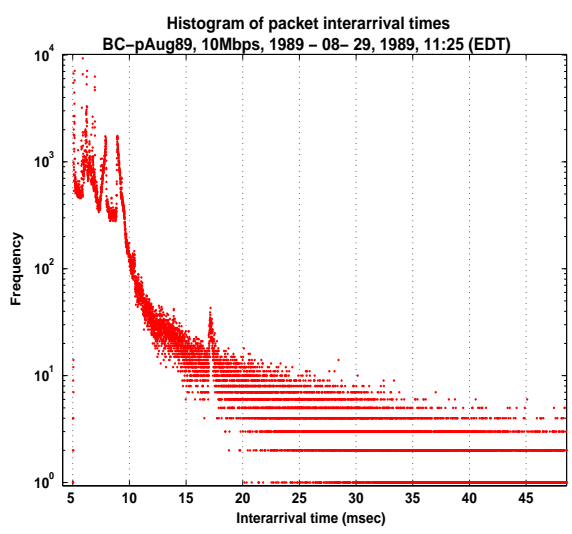

(c) Truncated histogram of packet interarrival times for the BC-pAug89 trace.

Fig. 2. Distributions of packet interarrival times for the WIDE, LBL and BC traces.

has rich infrastructure in Asia and Europe.

Throughout the paper, we will use these traces interchangeably. Although we will be presenting figures for some of the traces in each case, the results presented apply to all described traces irrespective of the direction of the link, unless otherwise specified.

\section{Traffic Appears Poisson at Sub-Second Time SCALES}

In this section, we show that the distribution of packet interarrival times of backbone traffic can be well described by an exponential distribution. Furthermore, packet sizes and interarrival times appear independent.

\section{A. Distribution of Packet Interarrival Times}

We study the interarrival distributions of all the backbone traces described in section III. We find that the packet interarrival time distribution is well approximated by an exponential distribution both for the OC48 traces, as well as for the WIDE backbone traces.

An interarrival time distribution consists of two portions [5]. One that can contain back-to-back packets and another for packets that are guaranteed to be separated by idle time. For heavily utilized links, interarrival times are function of packet sizes since many packets are sent back-to-back. For links that are overprovisioned, the distribution tends to contain most probability in the "idle" portion.

For the link in question, packet interarrival times can be closely approximated by an exponential distribution. The packet interarrival distributions for one of the MFN traces, for the aggregate (total), TCP and UDP traffic is shown in Fig. 1. The CCDF of packet interarrival times is a straight line when the $\mathrm{Y}$ axis is plotted in log scale, which corresponds to exponential distribution.

A closer look at the CCDF reveals that there is a trivial deviation from the exponential line at two points: the tail of the 

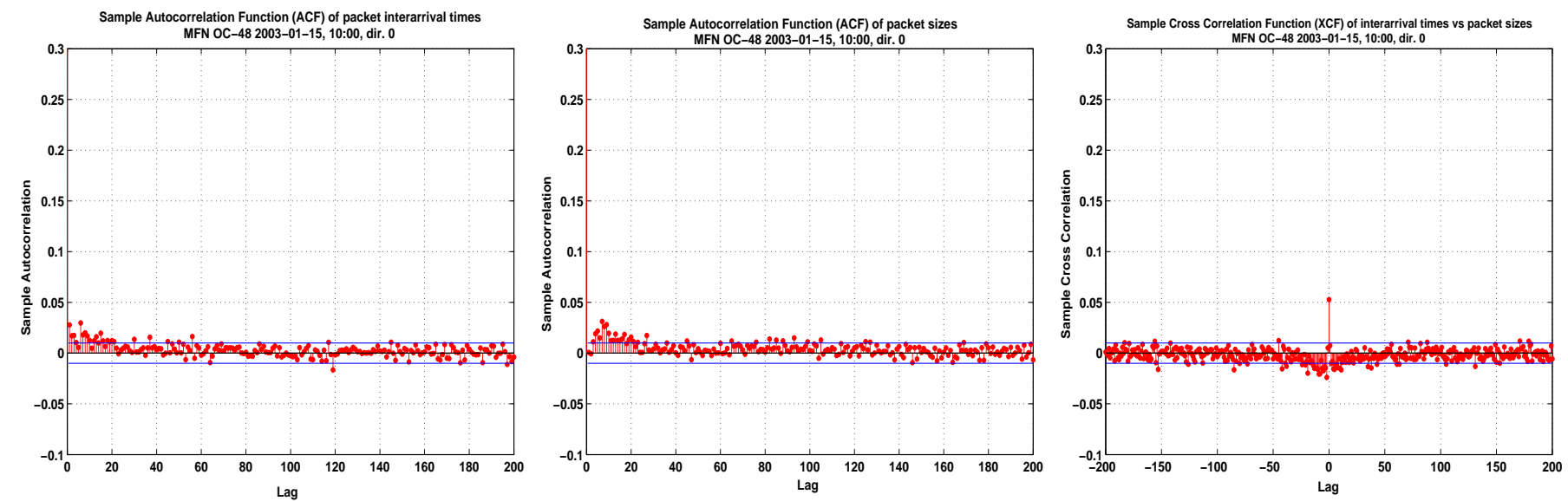

Fig. 3. Autocorrelation function of packet interarrival times and packet sizes and sample cross correlation. All the correlation coefficients are within the $95 \%$ confidence intervals except for a small number of coefficients for small lags.

distribution and for small interarrival times. The tail represents a minimum portion of the distribution (less than $10^{-6}$ ). On the other hand, the shape of the distribution for small values of interarrival times (less than $6 \mu s$ ) is the effect of back-to-back packets and Layer 2 technologies. However, linear least squares fitting shows that the CCDF is well described by an exponential with confidence $99.99 \%$. Similar observations hold for the interarrival time distribution of specific packet sizes (i.e., interarrival distribution of 1500-byte packets); interarrival times are exponentially distributed.

Nevertheless, exponential distribution for packet interarrival times is not specific only to our OC48 traces. Fig.2(a) shows the packet interarrival CCDF for a WIDE backbone trace (June 16, 2003). The distribution is qualitatively similar to the distributions of the OC48 traces. With $99.89 \%$ confidence the distribution is well described by the exponential distribution. Note that the bandwidth of the WIDE link is 100Mbps and despite the huge bandwidth difference with the OC48 link (2.5Gbps), both links are characterized by exponentially distributed interarrival times.

To highlight the differences between current backbone traces and past Ethernet-link traces, Fig. 2(b) and 2(c) show the CCDF and histogram of interarrival times for the LBL and BC traces. We present the CCDF of one of the traces and the histogram of the other, since both traces have similar characteristics. Their shapes are in agreement with distributions that have been observed in Ethernet traffic [18], [20] in the mid '80s and early '90s, before the identification of self-similarity. The spikes seen in the histogram (caused mostly by request-response protocols) result in the deviation of the CCDF at the early values of the distribution. Furthermore, the tail of the distribution is heavier than that of an exponential distribution indicating the effects of individual flow characteristics in these limited Ethernet links (10Mbps).

\section{B. Independence}

We separately examine and show independence of packet sizes and interarrival times of the OC48 link traces. The independence is validated using various tests: a) the autocorrelation (ACF) and cross-correlation (XCF) functions, b) the Box-Ljung statistic, c) the visual inspection of consecutive arrivals, and d) the conditional probabilities.

Correlation functions: Fig. 3 presents the autocorrelation and cross-correlation functions calculated for 200 lags for 40,000 consecutive packet arrivals for the packet sizes and interarrivals series. The sizes series consists of the actual packet sizes as individual packets arrive; the interarrivals series consists of the timestamp differences between consecutive packets. Apart from some limited correlation at small time lags, sizes and interarrivals are not correlated. The trivial correlation at small time lags close to zero indicated by correlation coefficients that are just outside the $95 \%$ confidence interval of zero (straight lines just above and below zero), is the effect of backto-back packets and phenomena that cause the interarrival distribution to deviate from the exponential for interarrival times less than $6 \mu s$.

Increasing the number of lags or the size of the series (number of packet arrivals in this case) does not have any effect on the ACF or XCF. However, nonstationarity can interfere with variance calculations (see sections $\mathrm{V}, \mathrm{VI}$ ) when the correlation is estimated across nonstationary time intervals. This is an artifact of the way the ACF and XCF functions are estimated. A change in the mean of the series will result in distant events in time to seem correlated according to the ACF or XCF, while they are not (recall the bug analogy from the introduction).

Box-Ljung statistic: The Box-Ljung statistic [30] $Q_{k}$ is defined as:

$$
Q_{k}=n(n+2) \sum_{i=1}^{k} \frac{r_{i}^{2}}{n-i},
$$

where $r_{i}$ is the autocorrelation coefficient for lags $1 \leq i \leq k$ 
and $n$ is the length of the series. The $Q_{k}$ statistic is compared with $\chi^{2}$ distribution with $k$ degrees of freedom in order to test the null hypothesis. For large values of $k$ the following approximation for the $\chi^{2}$ distribution with $k$ degrees of freedom can be used [27]:

$$
\chi_{k, 1-a}^{2} \approx k\left(1-\frac{2}{9 k}+z_{1-a} \sqrt{2 /(9 k)}\right)^{3},
$$

where $z_{1-a}$ is the upper percentile of the $N(0,1)$ distribution. We applied the test for lags 1 up to 200, for varying numbers of consecutive packet arrivals for both the interarrival times and packet sizes. The Box-Ljung statistic shows that both variables can be considered i.i.d with $95 \%$ confidence for up to a certain number of consecutive packet arrivals. Increasing the lag produces similar results. Furthermore, independence is valid for a large number of consecutive arrivals. The point where dependence appears differs with the trace and time within the trace. For interarrival times, independence holds for 20,000 consecutive packet arrivals on the average according to the test. For the packet sizes series the average is approximately 16,000 consecutive packet arrivals.

Consecutive arrivals: We visually examine size and interarrival time scatter plots of consecutive packet arrivals similar to Fig. 4. Although this type of figures does not prove independence, it can reveal dependencies in the dataset. The $\mathrm{X}$ axis shows the size of packet arrival $k$, while the $\mathrm{Y}$ axis shows the size of packet arrival $k+1$. Fig. 4 demonstrates that the plot is symmetric and no specific trends can be seen. At the end of $X$ and $\mathrm{Y}$ axes of the figure, we plot the histogram of packet sizes for reference.

Conditional probabilities: Examining the conditional probabilities of sizes and interarrivals also points to independence. We study the probabilities of sizes and interarrival times conditioned on the value of the previous size or interarrival time respectively. For example, each straight line in Fig. 5 presents the CCDF of packet interarrival times conditioned on the previous interarrival time being within seven different bins. Because the exponential distribution falls off rapidly with increasing time, the bin sizes increase with powers of 2 (i.e., $0-2 \mu s, 2-4 \mu s . .64-$ $128 \mu \mathrm{s}$ ). If the interarrival times were independent, the lines would fall on top of each other, as is the case for Fig. 5.

\section{Burst Sizes}

To stress-test the claim for the memoryless properties of Poisson arrivals and independence, we studied bursts of packets. A burst describes successive packet arrivals with interarrival times less than a default value, which is considered to be the idle period. However, in our traces the idle period cannot be identified by the interarrival distribution. Thus, in order to define bursts, we use different values of interarrival time. If the arrival process is memoryless, the characteristics of the burst should remain the same irrespective of the interarrival time that defines the idle period. We find that the distributions of the duration of the busy/idle period, as well as the number of packets

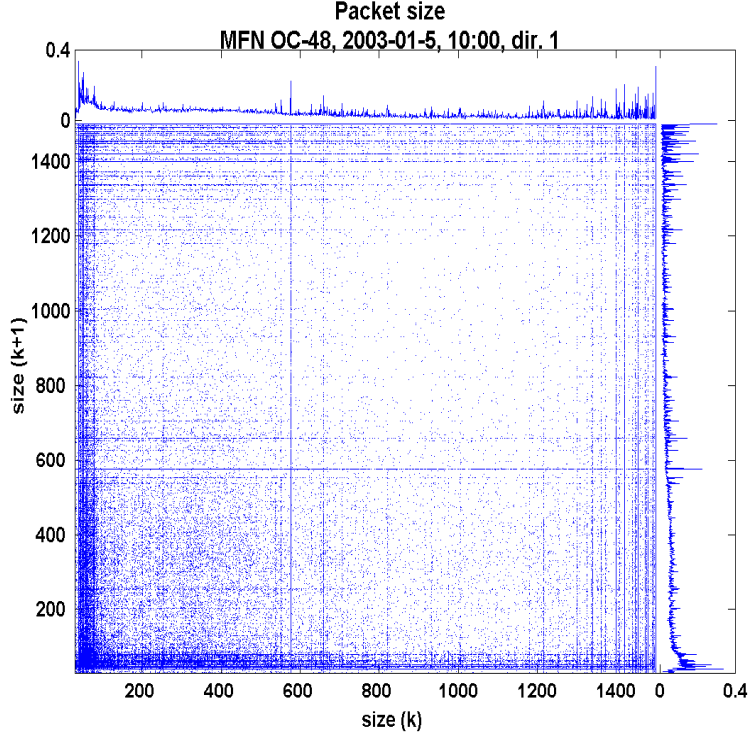

Fig. 4. Scatter plot for sizes of 1,000,000 consecutive packet arrivals. The figure is symmetric and does not show any patterns that would be indicative of dependence between sizes.

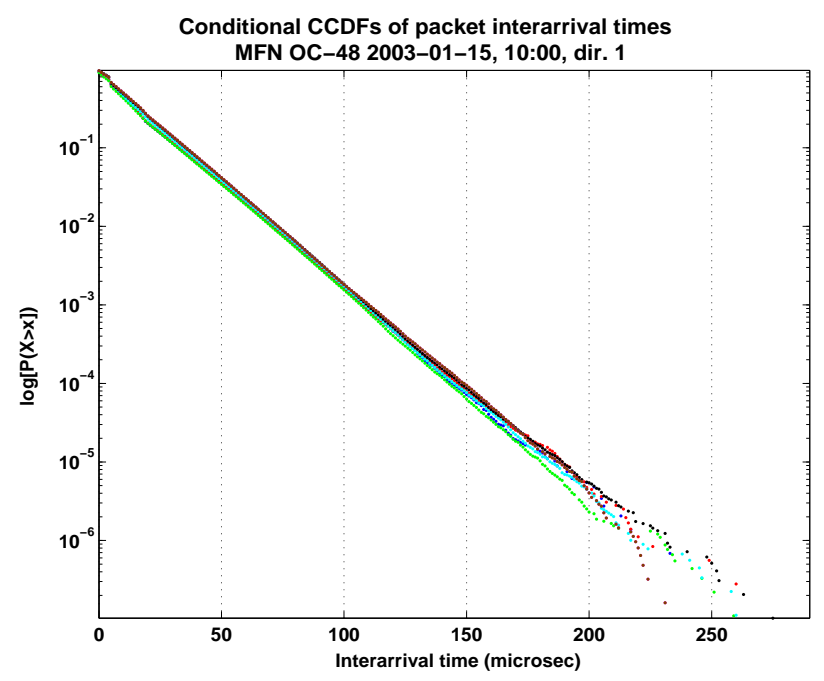

Fig. 5. CCDFs for interarrival times conditioned on the previous interarrival being in seven different bins based on powers of two.

or bytes in a busy period are well approximated by exponential distributions. This is irrespective of the interarrival time that is used as the boundary for distinguishing between idle and busy periods.

\section{TrafFic Appears Surprisingly Smooth, But Nonstationary, at Multi-Second Time Scales}

In this section, we demonstrate that Internet traffic is nonstationary. Furthermore, we discuss a number of possible causes leading to nonstationarity and characterize the behavior. 

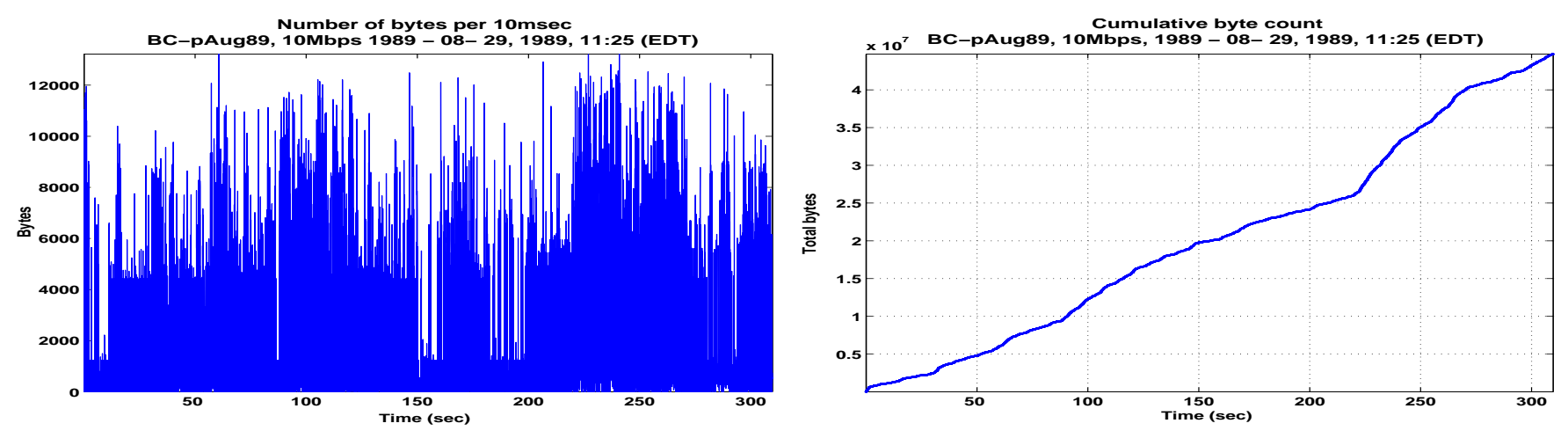

Fig. 6. Nonstationarity in $B C$-pAug89 trace file. LEFT: The byte count during consecutive $10 \mathrm{msec}$ intervals varies chaotically over a 6 minute window. RIGHT: The same data presented as cumulative bytes sent as a function of time looks surprisingly smooth, although clearly nonstationary.

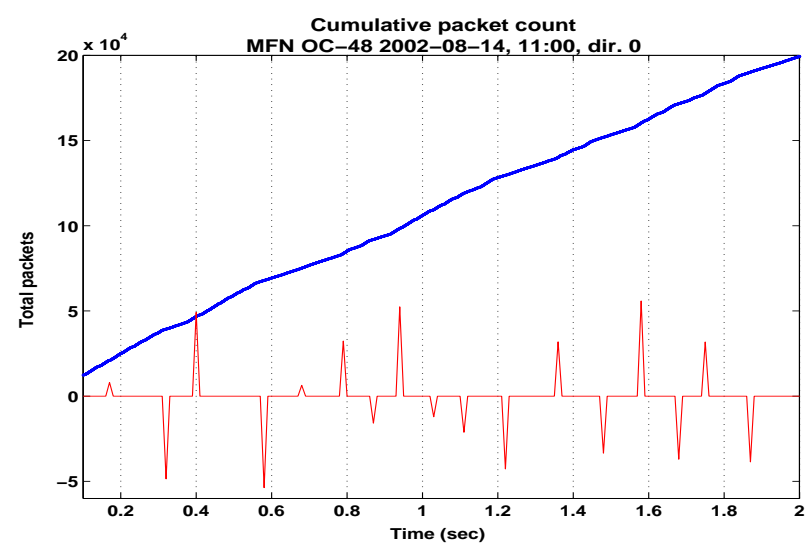

Fig. 7. The cumulative number of packet arrivals can be seen to change in an approximately piecewise-linear manner. The figure shows $2 \mathrm{sec}$ worth of traffic.

\section{A. Traffic has become smoother over time.}

Fig. 6 displays approximately 6 minutes of data from the well-known BC-pAug89 packet trace dataset [1], [28] in terms of both the familiar image of chaotically-varying byte counts per $10 \mathrm{msec}$. interval, as well as a cumulative display of total bytes sent as a function of time without using any data aggregation or smoothing. The smoothness of the cumulative graph (Fig. 6, right) is striking, in comparison to the more familiar chaotically varying appearance of the per-interval graph (Fig. 6, left). In particular, we see that despite the burstiness of individual packet arrivals, the slope of the cumulative traffic curve is well defined for time scales on the order of seconds. Although the slope may remain relatively constant for several minutes at a time, it is clearly a time-varying function.

Fig. 7 shows that current Internet backbone traffic exhibits similar nonstationarity. In Fig. 7 we show the cumulative number of packets sent over a 2 second interval from the August 2002 dataset. Notice that the total number of individual data points in each curve is approximately the same, since the changes in link speed (2.5 Gbps vs. 10Mbps) and measurement period ( $2 \mathrm{sec}$. vs. 6min.) between these two figures are complementary. However, the slope of the new dataset is significantly smoother than the earlier graph, and shows a distinctive pattern of piecewise linear segments separated by well-defined corners.

The spikes shown near the bottom of Fig. 7 highlight the points at which the rate changes. The height and direction of each spike represents the relative magnitude of change in the slope. In order to find the change points, a one-dimensional version of the Canny Edge Detector algorithm is applied [6]. The algorithm is often used in image processing to reveal object boundaries. The algorithm works as follows: first, Gaussian filtering is applied to the time-series to filter out high frequency noise. Then, the point where the gradient (i.e., derivative) reaches a peak value (i.e., a "ridge line") is found. The ridge lines are finally refined by setting to zero all points that are on the sloping edges leading up to the peak, but not actually there.

\section{B. Possible causes for nonstationarity.}

Among the overwhelming number of studies documenting self-similarity and long-range dependence in Internet traffic, a few authors have identified various examples of nonstationarity. In [45] [46], for example, the authors examine different notions of stationarity for various end-to-end performance parameters of network traffic. It is suggested that the notion of stationarity depends on the scale of observation. Moreover, the authors show that many processes (e.g., loss episodes) can be well modeled as i.i.d within change free regions, where stationarity is assumed. This concept of describing the overall network behavior as a series of piecewise-stationary intervals seems equally applicable to our traffic data. Thus, in the remainder of this section, we speculate on several possible mechanisms that could be responsible for creating the piecewise-stationary traffic patterns we have observed in the data.

Clearly, the simplest possible explanation for nonstationarity is the variation of the number of active sources over time. This 


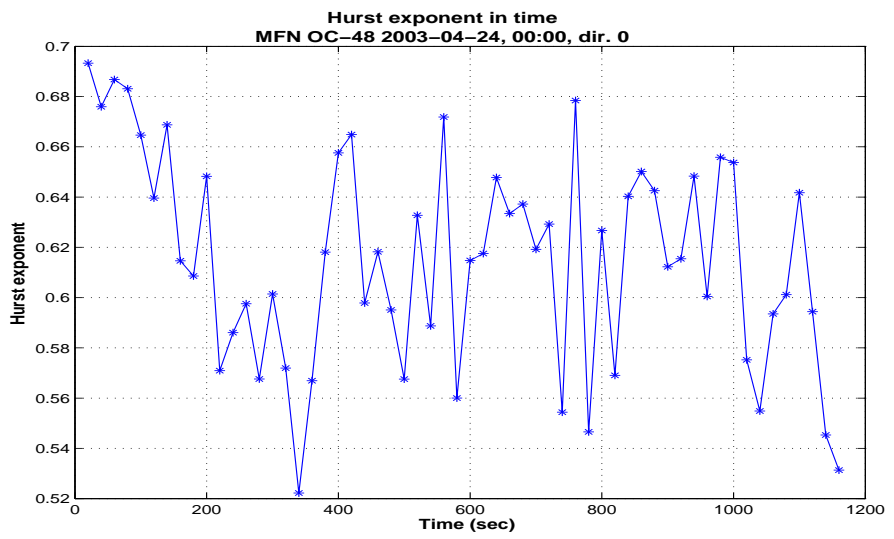

(a) Nonstationarity of the Hurst Exponent for small time scales across disjoint $20 \mathrm{sec}$ intervals (each of which contains approximately 2 million data points).

Fig. 8. Variation of Hurst exponent with time and index of dispersion (IDI)

is obviously responsible for (large) diurnal traffic variations, and the authors in [7] [8] suggest that it may also be a significant factor over much shorter time periods until the number of multiplexed sources becomes large enough to drive the arrival process to Poisson and sizes to independent. Although this explanation could certainly apply to links that support a relatively small number of multiplexed sources (e.g., LAN segments similar to the well-known BC-pAug89 trace), it seems far less reasonable as a possible explanation for the piecewise-linear variations we found in our OC-48 traces because the number of individual flows that were both large and fast enough to create visible rate changes at these scales was extremely small.

Another obvious possibility is to consider self-similarity in the traffic generation process. In this case, we must try to distinguish between nonstationarity and long-range dependence, since it is well known that LRD estimators can be fooled by nonstationary behavior (such as trends or periodicity). For this reason, the authors in [28] suggest partitioning the time-series into disjoint segments and separately calculating the value of the Hurst exponent for each segment. We applied the same procedure to our OC48 traces by partitioning one of the 20 minute traces to form 60 disjoint time-series, each containing approximately 2 million samples from $20 \mathrm{sec}$ intervals. The results of our analysis are shown in Fig. 8(a), which shows that the Hurst exponent value varies significantly over time, oscillating between 0.5-0.7 for small scales. Similar observations hold for the case of larger scales where the Hurst exponent value varies between 0.65-0.9. This suggests that our current Internet core traces are nonstationary, at least for time scales on the order of approximately one hour.

We also considered the possibility that routing changes might be responsible for the variations in the piecewise linear traffic rates. Note that a routing change could affect the measured traffic on our link either directly, by inserting or removing our measurement link from the paths followed by a (set of) active

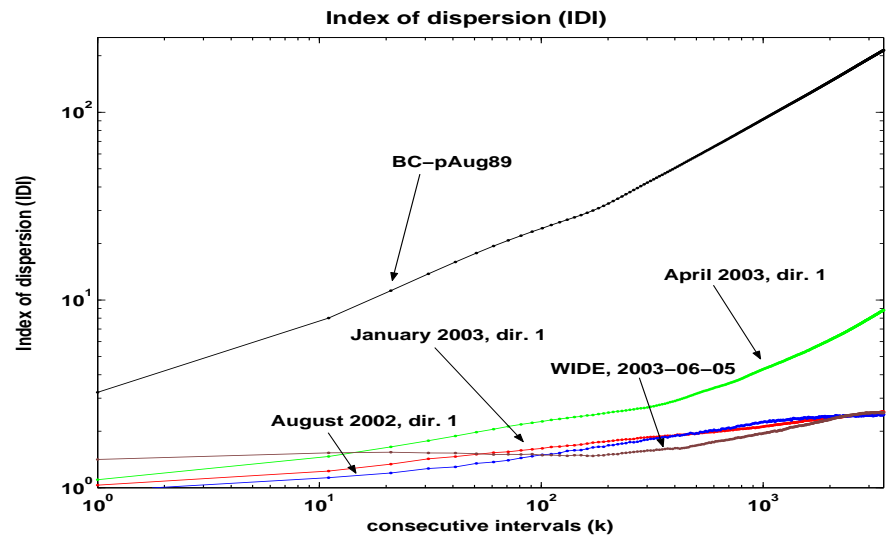

(b) Index of dispersion for intervals (IDI) for blocks of $k$ consecutive packet interarrival times obtained from multiple trace files. flow(s), or indirectly, by inserting or removing other traffic from a distant link and hence triggering a change in rate for the active flow(s) that use both links. This would be especially true for highly reactive TCP streams that suddenly see more bitrate available [38]. According to the routing persistence results reported in [46], approximately $10 \%$ of the commercial Internet routes had lifetimes of a few hours or less. Moreover, their cumulative distribution function for route lifetimes (based on sampling more than 36,000 host-pairs) was very flat across short time scales. Thus, since routing update protocols are specifically designed to avoid synchronization in the update times, and since the entire Internet contains a huge number of routers, we cannot reject the possibility that the mean time between routing updates of both types visible to our measurement point is below one second. This hypothesis will be left as the subject of further study.

However, perhaps the most convincing explanation for this type of nonstationary behavior comes from [39], where the authors carefully develop a methodology for quantifying the deviation from the Poisson limit of an aggregated arrival process composed of large numbers of highly-variable individual streams, when viewed over a wide range of different time scales. In particular, they show how aggregation of sources can "transfer" variability (which originated from the stationary, high-variance packet interarrival time distribution representing a single source) to the aggregate arrival process (which quickly takes on the characteristics of an almost-perfect Poisson process with a time-dependent mean arrival rate). Consequently, they show how the aggregate arrival process can behave like Poisson process in conjunction with small buffer sizes, but not with large buffer sizes, because of the cumulative variability caused by large numbers of small long-term covariance terms. In other words, these many small deviations from the Poisson limit may occasionally align themselves in peculiar ways to create artifacts that are visible across large time scales, similar to 

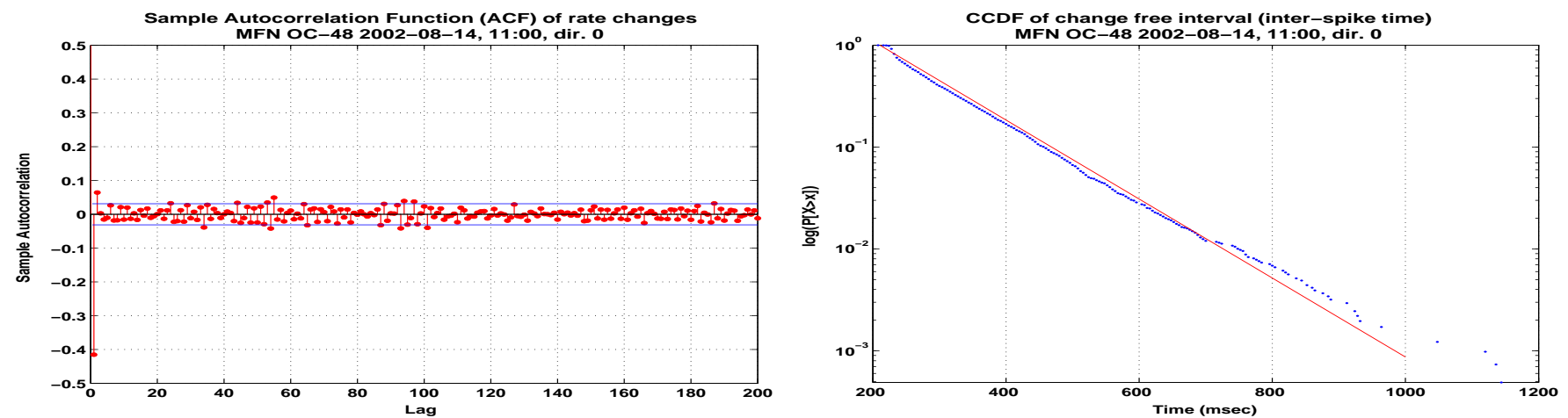

Fig. 9. Characterizing the piecewise-linear structure of the data. LEFT: Autocorrelation function for the magnitude of the rate change sequence falls within the $95 \%$ confidence window of zero. RIGHT: The CCDF for the distribution of the length of the change free intervals follows the exponential distribution.

the beats produced by two tuning forks set to nearly identical frequencies.

Such deviations can be visualized using the index of dispersion for intervals (IDI) [39], which is defined as the sequence $\left\{c_{k}^{2}\right\}, k \geq 1$, where:

$$
c_{k}^{2}=\frac{k \operatorname{Var}\left(S_{k}\right)}{\left[E\left(S_{k}\right)\right]^{2}}
$$

and the random variable $S_{k}$ is the sum of $k$ consecutive interarrival times. ${ }^{2}$ Notice that if the arrival process is an ideal Poisson process, then we should have $c_{k}^{2} \equiv 1$ for every $k$. However, if the arrival process has higher variance at some time scale, then $c_{k}$ will tend to increase as a function of $k$.

Fig. 8(b) shows how $c_{k}^{2}$ varies as a function of $k$ for a variety of trace datasets. Similar to Fig. 4 from [39], we expect the Poisson assumption to be quite effective over short ranges (so that $c_{k}^{2} \approx 1$ for small values of $k$ ), but to gradually degrade as $k$ increases. For example, the authors in [39] found that when aggregating 60 voice sources (each having a squared coefficient of variation of 18.1), the Poisson assumption was very good for arrival sequences of length less than approximately 50, beyond which $c_{k}^{2}$ began to increase almost linearly. Notice that the well-known BC-pAug89 trace is a very poor fit to the Poisson distribution, but the recent traces obtained from both our OC-48 link and the $100 \mathrm{Mbps}$ trans-Pacific link monitored by the WIDE project show a remarkably good fit, even for large block sizes. Except for the April 2003 trace, all of our recent traces fit the Poisson assumption very well even when we increase the arrival sequence lengths into the thousands. Note

\footnotetext{
${ }^{2}[39]$ includes the following remark about the IDI. "This technique applies much more broadly, and we believe that it can greatly help understand other complex arrival processes in queueing systems (and elsewhere)." We agree, and would further like to emphasize its advantage of normalizing the autocovariances across the interarrival time sequence, $\left\{X_{i}\right\}$, with respect to $E[X]$ rather to $\operatorname{Var}(X)$. Hence the magnitude of $c_{k}^{2}$ is a meaningful quantity, which allows us to determine the actual significance of the correlations across a distance of $k$ steps. In contrast, the autocorrelation function, $\rho(k)$, is normalized by the standard deviation, $\sigma \equiv \rho(0)$, so we can only compare the relative significance of correlations across different distances - without knowing whether any of these deviations are significantly greater than zero.
}

that, $c_{k}^{2}$ does not depend on the specific part of the trace but it does depend on the length of the series used. That is, its value remains approximately the same when estimated at any disjoint piece of the trace with the same number of points. However, when the number of samples in the series is increased past the point where more than 5-10 seconds are represented (e.g., more than $500,000-2,000,000$ consecutive arrivals depending on the OC48 traces), it increases due to the piecewise nonstationarity. For Fig. 8(b), we used 500,000 arrivals to estimate IDI.

\section{Characterizing the nonstationarity.}

To quantify the behavior of the aggregate traffic process over longer time scales, we studied both the magnitude of the rate change events that separate each interval (i.e., the height of the "spikes" in Fig. 7) and the durations of the piecewise-linear change free intervals between each "spike". Our results are shown in Fig. 9. We found that the magnitude of the rate change events appears to be uncorrelated beyond a significant negative correlation at distance one. This negative correlation at lag one implies that an increase in rate is followed by a decrease. This is also evident from the alternation of direction of changes in Fig.7. However, the remainder of the ACF is very flat and falls within the $95 \%$ confidence interval of zero. We also found that the durations of the change free intervals follow the exponential distribution. Although we do not show it here because of space limitations, the ACF for the durations of the change free intervals fell within the $95 \%$ confidence interval of zero.

These results are consistent with theoretical results on the aggregation of large numbers of independent renewal streams under the intermediate connection rate model in [17], where we increase the number of active sources in proportion to the available service rate to maintain a constant normalized load. They show that the variability of the aggregate arrival process converges to a non-stable, non-Gaussian process with a zero mean and stationary increments ${ }^{3}$. Thus, by combining our ob-

\footnotetext{
${ }^{3}$ In [17], they also show that the limit process is continuous, has finite moments of all orders, is second-order self-similar, but not self-similar.
} 


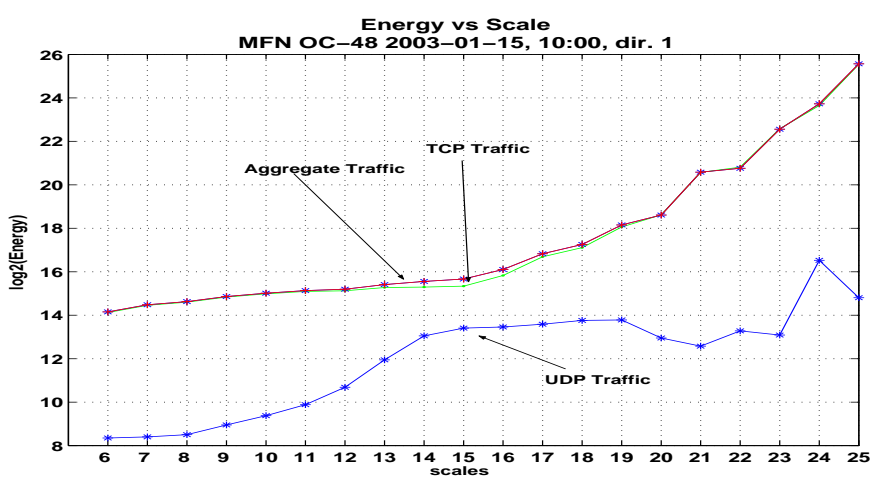

(a) The Hurst exponent is approximately 0.6 at small scales and 0.8 at larger scales for the aggregate and TCP traffic.

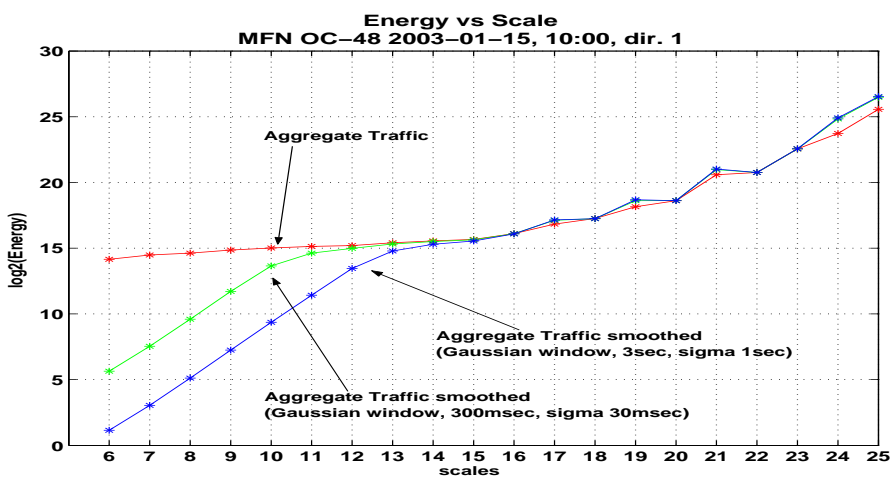

(b) The scaling behavior of the original and smoothed aggregate traffic. At large scales the behavior is the same.

Fig. 10. Wavelet (Haar) energy versus scale for byte counts. (time $=2^{\text {scale }} * 10 \mu s$.)

TABLE II

ScAle of Change for the Hurst exponent value.

\begin{tabular}{|c||c|c|c|}
\hline & August 2002 & January 2003 & April 2003 \\
\hline \hline Dir. 0 & $650 \mathrm{msec}$ & $40-80 \mathrm{msec}$ & $320 \mathrm{msec}$ \\
\hline Dir. 1 & $80-320 \mathrm{msec}$ & $160-320 \mathrm{msec}$ & $320 \mathrm{msec}$ \\
\hline
\end{tabular}

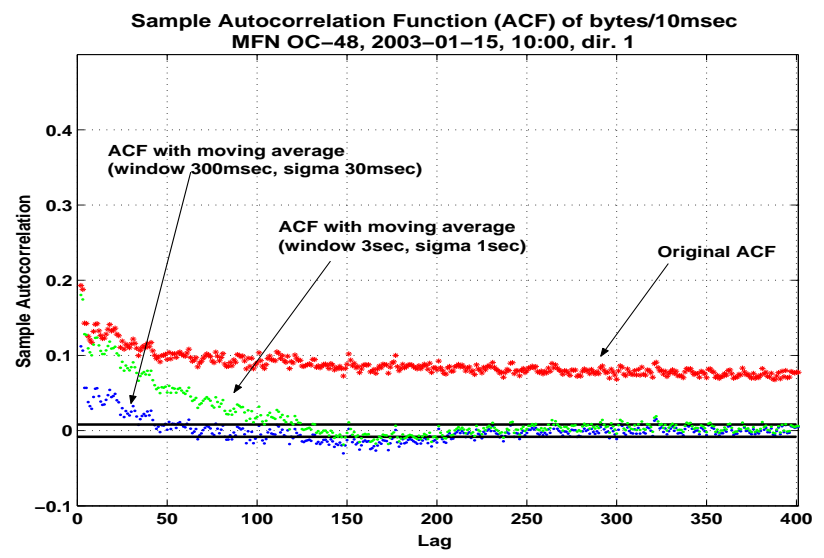

Fig. 11. ACF calculated with global mean and moving average mean based on a Gaussian window. Correlation coefficients drop to zero after first lags when local nonstationarities are removed with moving average.

servations with these theoretical results, we conjecture that the nonstationarity of the Poisson traffic rate may be well described by a Markovian random walk model.

\section{TRaffic Appears LRD at Large Time Scales}

In this section, we study the scaling behavior of the backbone traffic using the same set of OC48-link traces from which we showed nonstationary Poisson behavior at multi-second time scales. Our observations across large time scales show that backbone traffic demonstrates long-range dependence as measured by the Hurst exponent estimators. The scaling behavior is characterized by a dichotomy between small and large scales. To analyze the scaling behavior, we study the series of byte and packet counts with smallest aggregation level at $10 \mu \mathrm{s}$. To overcome accuracy related problems of the Hurst exponent estimators [26] [32] [22] [40], all common estimators [4] [40] [3] are applied to each series.

Our analysis shows that backbone traffic is characterized by long-range dependence. However, the intensity of correlation depends on the scale of observation. Specifically, in all traces analyzed we observe a dichotomy in the scaling in agreement with previous studies [16] [15] [47]. The point of change is within the millisecond scales, albeit different for each case. However, the pattern is the same: At scales below the point of change the Hurst exponent is just above 0.6, while at larger scales it varies between 0.7 and 0.85 depending on the trace and the estimator used ${ }^{4}$. Table II summarizes the points of change for the value of Hurst exponent. Similar observations hold for the packet counts case.

Fig. 10(a) presents the scaling behavior of byte counts using the wavelet estimator for the aggregate, TCP and UDP traffic. The base wavelet used is the Haar wavelet for scales 6-25 (640 $\mu s-5 \mathrm{~min})$. Because of the dominance of the TCP traffic (Table I), the energy line for the UDP traffic appears lower in both figures. Fig. 10(a) demonstrates that the scaling behavior of the aggregate traffic is highly correlated with the behavior of TCP traffic. Both show the change in the value of the Hurst from 0.62 to 0.8 at the same scale (16). On the contrary, UDP scaling behavior does not follow the pattern of the aggregate traffic or TCP traffic.

In order to highlight the thin line between long-range dependence and nonstationarity, we offer the following example motivated by our observations in the previous sections. We calculated the ACF coefficients for 400 lags using two different ways: a) The regular ACF function described in section II and

\footnotetext{
${ }^{4}$ Figures showing the result of the Hurst exponent estimators for our traces have been omitted due to space limitations. However all show with $99 \%$ confidence similar dichotomy and Hurst exponent values.
} 
b) replacing the global average used in the ACF, by a moving average (calculated using a Gaussian window), thus removing local nonstationarities from the calculation of the autocorrelation function. Fig. 11 shows the effect of removing "nonstationarity" from the January OC48 trace on the autocorrelation function. The correlations fall within the $95 \%$ confidence interval of zero, after $1 \mathrm{msec}$ when the moving average is used. The magnitude of the correlation depends mostly on the standard deviation (sigma) of the Gaussian window, and also on its size.

On the other hand, when the Hurst exponent estimators are applied to the Gaussian moving average function, we find that the smoothed function has the same Hurst exponent (approximately 0.8 ) as the original series at large enough scales. Fig. 10(b) presents the scaling behavior for the aggregate traffic after the Gaussian smoothing. Because high frequency noise has been removed, the smoothed curves have lower energy at small scales. On the other hand, high frequency noise is present in Fig. 11, where there are only deviations from the local average. At larger scales, the scaling behavior is the same as the original aggregate traffic (Fig. 10(b), beyond scale 13).

However, since this same Gaussian moving average function can also be thought of as an approximation to the piecewise linear rate function described in the section $\mathrm{V}$ with its corners rounded off, we now have two different perspectives of the same nonstationarity: exponentially distributed, stationary uncorrelated increments and long-term correlations ${ }^{5}$. While this description of the rates series is in agreement with fractional Gaussian noise theory, this is clearly a question for further study in the future!

\section{CONCLUSIONS}

In this paper, we revisit the validity of the Poisson assumption by examining a number of current and historical traces of Internet traffic. We find that at sub-second time scales, backbone traffic appears to be well described by Poisson packet arrivals. Our study provides evidence for how the ongoing pattern of Internet evolution may potentially affect the future characteristics of its traffic, as shown by our traces. We conjecture that the particular way in which this increase in scale is unfolding seems to be pushing the Internet in the general direction of easier-to-understand and better-behaved traffic models (i.e., the Poisson assumption) - or at least not in the direction of sophisticated traffic models!

More specifically based on traces from the MFN and WIDE backbones, we found that up to sub-second time scales traffic is well characterized by a stationary Poisson model. This is important because it covers the relevant time scales for the delivery of a single packet (i.e., the Round-Trip Time). Beyond that point, the traffic seems to take on a distinctive form of nonstationary behavior, which consists of short intervals of "change free regions" punctuated by sudden change events. The

\footnotetext{
${ }^{5}$ Note that in section $\mathrm{V}$ we examined the rate changes, while the Gaussian moving average function corresponds to the series of the rates themselves.
}

durations of the change free intervals were found to be exponentially distributed and uncorrelated, while the change events themselves appeared to be stationary with only a trivial onestep (negative) correlation in the increments. We note that these observations are also consistent with the theoretical results for large-scale aggregations of renewal processes which have been derived under the assumption of scaling the number of sources and network capacity together to keep the normalized offered load fixed. We also show that this type of traffic model (i.e., Poisson with nonstationarity at multi-second time scales) is consistent with the kind of long-range dependence that is commonly observed in network data over larger time scales.

It would be interesting to analyze more data traces from: a) other backbone links, and b) links towards the periphery of the network. It could very well turn out that different links exhibit different behavior especially at small time scales as suggested by some interesting recent studies [21] [47]. Scaling phenomena especially at small time scales may be sensitive to the traffic mix in terms of applications and the idiosyncrasies of low level protocols.

Our work has also left a number of interesting questions unanswered, which remain as subjects for further study. Most importantly, is the type of nonstationary behavior we see at multi-second time scales sufficient to explain everything, or are there even-larger scale effects remaining to be discovered? Another important open issue is finding the mechanism that is responsible for the distinctive piecewise-linear variation in the rate.

Finally, we found that focusing on the proper time scale turned out to be a recurring theme in our work as well as in many of the references cited. Just as the analogy of the two bugs in the garden shows it is important to avoid excessively large scales, we must also be careful not to focus on too small a time scale. Although Whitt pointed out that the right time scale must be an increasing function of load placed on a network resource [39], Norros [33] has observed that network traffic sources have the flexibility and intelligence to adapt their transmission policies to the resources currently available in the network. Thus, we conjecture that the traffic characteristics for the Internet backbone may continue to grow even better behaved in the future.

\section{ACKNOWLEDGMENTS}

We would like to thank Constantinos Dovrolis and Mark Crovella for their valuable comments and constructive criticism. Thanks are also due to kc claffy and Margaret Murray for their support. 


\section{REFERENCES}

[1] Internet Traffic Archive. http://ita.ee.lbl.gov/.

[2] MAWI Working Group Traffic Archive. http://tracer.csl.sony.co.jp/mawi/.

[3] P. Abry and D. Veitch. Wavelet Analysis of Long-Range Dependence Traffic. In IEEE Transactions on Information Theory, 1998.

[4] J. Beran. Statistics for Long-memory Processes. Chapman and Hall, New York, 1994.

[5] A. Broido, R. King, E. Nemeth, and kc claffy. Radon Spectroscopy of Packet Delay. In Proceedings of the 18th International Teletraffic Congress, Berlin, Aug.-Sep.2003.

[6] J. Canny. A Computational Approach To Edge Detection. In IEEE Transactions Pattern Analysis and Machine Intelligence, 1986.

[7] J. Cao, W. Cleveland, D. Lin, and D. Sun. Internet Traffic Tends to Poisson and Independent as the Load Increases. Bell Labs Technical Report, 2001.

[8] J. Cao, W. Cleveland, D. Lin, and D. Sun. On the Nonstationarity of Internet Traffic. SIGMETRICS/Performance, 2001.

[9] K. Cho, K. Mitsuya, and A. Kato. Traffic Data Repository at the WIDE Project. Proceedings of USENIX 2000 FREENIX Track, San Diego CA, June 2000.

[10] M. E. Crovella and A. Bestavros. Self-Similarity in World Wide Web Traffic: Evidence and Possible Causes. In IEEE/ACM Transactions on Networking, 1997

[11] N. G. Duffield, J. T. Lewis, N. O'Connell, R. Russel, and F. Toomey. Statistical issues raised by Bellcore data. In IEE 11'th UK Teletraffic Symposium, March 1994.

[12] N.G. Duffield, J.T. Lewis, N. O'Connell, R. Russell, and F. Toomey. Predicting Quality of Service for Traffic with Long-Range Fluctuations. In IEEE International Conference on Communications, Seattle, 1995.

[13] Endace. http://www.endace.com/.

[14] A. Erramilli, P. Pruthi, and W. Willinger. Self-Similarity in High-Speed Network Traffic Measurements: Fact or Artifact. In VTT symposium, volume 154, pages 299-310, 1995.

[15] A. Feldmann, A. C. Gilbert, P. Huang, and W. Willinger. Dynamics of IP Traffic: A Study of the Role of Variability and The Impact of Control. In SIGCOMM, pages 301-313, 1999.

[16] A. Feldmann, A. C. Gilbert, W. Willinger, and T. G. Kurtz. The Changing Nature of Network Traffic: Scaling Phenomena. In ACM Computer Communication Review, volume 28, pages 5-29, 1998.

[17] R. Gaigalas and I. Kaj. Convergence of Scaled Renewal Processes and a Packet Arrival Model. In Journal of the Bernoulli Society for Mathematical Statistics and Probability, (to appear).

[18] R. Gusella. A Measurement Study of Diskless Workstation Traffic on an Ethernet. In IEEE Transactions on Communications, volume 38, September 1990.

[19] N. Hohn, D. Veitch, and P. Abry. Cluster Processes, a Natural Language for Network Traffic. IEEE Transactions on Networking, 2003.

[20] R. Jain and S. Routhier. Packet Trains: Measurements and a New Model for Computer Network Traffic. In IEEE J. Select. Areas Commun., volume 4, pages 1162-1167, 1986.

[21] H. Jiang and C. Dovrolis. Source-level IP packet bursts: causes and effects. In ACM Internet Measurements Conference (IMC), Miami FL, 2003.

[22] T. Karagiannis, M. Faloutsos, and R.H. Riedi. Long-Range dependence:Now you see it, now you don't! In IEEE GLOBECOM, Global Internet Symposium, TAIPEI,2002.

[23] S. Karlin and H. Taylor. A First Course in Stochastic Processes, 2/e. Academic Press, New York, 1975.

[24] L. Kleinrock. Queueing Systems, Vol. II Computer Applications. Wiley, New York, 1976.

[25] W. Kramer, P. Sibbertsen, and C. Kleiber. Long Memory vs. Structural Change in Financial Time Series. http://www.sfb475.uni-dortmund.de/berichte/tr37-01.ps.

[26] M. Krunz. On the Limitations of the Variance-Time Test for Inference of Long-Range Dependence. In IEEE INFOCOM, 2001.

[27] A. M. Law and D. W. Kelton. Simulation Modeling and Analysis, 3/e, pages 418-419. 2000.

[28] W. E. Leland, M. S. Taqqu, W. Willinger, and D. V. Wilson. On the Self-Similar Nature of Ethernet Traffic. In IEEE/ACM Transactions on Networking, 1994
[29] M. Liljenstam and A. T. Ogielski. Crossover Scaling Effects in Aggregated TCP Traffic with Congestion Losses. In ACM Computer Communication Review, 2002.

[30] G. Ljung and G.Box. On a Measure of Lack of Fit in Time Series Models. In Biometrika '65, pages 297-303, 1978.

[31] J. Micheel, S. Donnelly, and I. Graham. Precision Timestamping of Network Packets. In Proceedings of ACM Sigcomm Internet Measurement Workshop (IMW), Nov 2001.

[32] S. Molnar and T. D. Dang. Pitfalls in Long Range Dependence Testing and Estimation. In GLOBECOM, 2000

[33] I. Norros. On the Use of Fractional Brownian Motion in the Theory of Connectionless Networks. In IEEE J. Select. Areas Commun., volume 13, pages 953-962, 1995.

[34] K. Park, G. Kim, and M.E.Crovella. On the Relationship Between File Sizes Transport Protocols, and Self-Similar Network Traffic. In Inter Conf. on Network Protocols, pages 171-180, Oct 1996.

[35] K. Park and W. Willinger. Self-similar network traffic: An overview. In Self-Similar Network Traffic and Performance Evaluation. WileyInterscience, 2000.

[36] V. Paxson and S. Floyd. Wide Area Traffic: The Failure of Poisson Modeling. IEEE/ACM Transactions on Networking, 1995.

[37] R. H. Riedi, M. S. Crouse, V. J. Ribeiro, and R. G. Baraniuk. A Multifractal Wavelet Model with Application to Network Traffic. IEEE Special Issue on Information Theory, pages 992-1018, 1999.

[38] S. Sarvotham, R. Riedi, and R. Baraniuk. Network Traffic Analysis and Modeling at the Connection Level. IEEE/ACM SIGCOMM, IMW, 2001.

[39] K. Sriram and W. Whitt. Characterizing Superposition Arrival Processes in Packet Multiplexors for Voice and Data. In IEEE J. Select. Areas Commun., volume 4, pages 833-846, 1986.

[40] M. S. Taqqu and V. Teverovsky. On Estimating the Intensity of LongRange Dependence in Finite and Infinite Variance Time Series. In A Practical Guide to Heavy Tails: Statistical Techniques and Applications, pages 177-217. Birkhauser, Boston, 1998.

[41] X. Tian, J. Wu, and C. Ji. A Unified Framework for Understanding Network Traffic Using Independent Wavelet Models. In IEEE INFOCOM, 2002.

[42] A. Veres and M. Boda. The Chaotic Nature of TCP Congestion Control. In IEEE INFOCOM, pages 1715-1723, 2000.

[43] A. Veres, Z. Kenesi, S. Molnar, and G. Vattay. On the Propagation of Long-range Dependency in the Internet. In SIGCOMM, 2000.

[44] W. Willinger, M. S. Taqqu, R. Sherman, and D. V. Wilson. Self-similarity through High-Variability: Statistical Analysis of Ethernet LAN Traffic at the Source Level. IEEE/ACM Transactions on Networking, 5(1):71-86, 1997.

[45] Y. Zhang and N. Duffield and V. Paxson and S. Shenker. On The Constancy of Internet Path Properties. In ACM SIGCOMM Internet Measurement Workshop, 2001.

[46] Y. Zhang and V. Paxson and S. Shenker. The Stationarity of Internet Path Properties: Routing, Loss, and Throughput. ACIRI Technical Report, 2000.

[47] Z. L. Zhang, V. Ribeiro, S. Moon, and C. Diot. Small-Time Scaling Behaviors of Internet Backbone Traffic: An Empirical Study. In IEEE INFOCOM, SF, 2003. 